\title{
Diabetics show Accelerated Progression of Knee Cartilage and Meniscal Lesions: Data from the Osteoarthritis Initiative
}

\author{
Jan Neumann ${ }^{1}$, Julio B. Guimaraes ${ }^{1}$, Ursula Heilmeier ${ }^{1}$, Gabby B. Joseph ${ }^{1}$, Michael C. \\ Nevitt $^{2}$, Charles E. McCulloch ${ }^{2}$, and Thomas M. Link ${ }^{1}$ \\ ${ }^{1}$ Musculoskeletal Quantitative Imaging Research Group, Department of Radiology \& Biomedical \\ Imaging, University of California San Francisco, San Francisco, CA USA \\ ${ }^{2}$ Department of Epidemiology and Biostatistics, University of California San Francisco, San \\ Francisco, CA USA
}

\section{Abstract}

Purpose.-To investigate whether subjects with diabetes show accelerated knee joint structural degeneration over 4 years compared to diabetes-free controls.

\begin{abstract}
Methods and Materials.-Two hundred forty-four participants with diabetes were selected from the Osteoarthritis Initiative cohort and matched with 244 diabetes-free controls. 3.0T MRI scans of the right knee were obtained at baseline and 4-year follow up. Evaluation of structural knee abnormalities was performed using the Whole-Organ Resonance Imaging Scoring system (WORMS). Linear regression analysis was conducted to compare structural temporal changes in each compartment, as well as the mean across all compartments by diabetes status.
\end{abstract}

Results-Study groups were similar in age ( 63.0 vs 63.3 years, $\mathrm{p}=0.73$ ), body mass index (31.5 vs $31.0 \mathrm{~kg} / \mathrm{m}^{2}$, p=0.21), sex (female $52.0 \%$ vs $52.9 \%$, $\mathrm{p}=0.85$ ) and radiographic Kellgren/ Lawrence score distribution ( $\mathrm{p}=0.99$ ). Structural degeneration was significantly worse in the knees of diabetics with an increase in the overall WORMS sum score (delta WORMS [95\% CI]: 4.87 [4.17,5.57], vs 3.23 [2.60,3.85] $\mathrm{p}=0.001)$. Moreover, diabetics showed a greater increase in cartilage lesions in the global knee $(\mathrm{p}<0.001)$, but also separately in the patella, lateral tibia, and both femoral compartments (lowest $\mathrm{p}$-value; $\mathrm{p}=0.001$ ). Furthermore, diabetics showed also a greater increase in meniscus lesion score, in both the medial $(\mathrm{p}=0.01)$ and lateral meniscus $(\mathrm{p}=0.01)$.

Conclusion: Diabetics exhibited a significantly greater increase in cartilage and meniscus lesions when compared to diabetes-free controls over 4 years. Overall, our findings suggest that diabetics exhibit a stronger deterioration of knee structure and are therefore potentially at higher risk for developing knee OA.

Corresponding author address: Jan Neumann, M.D., Department of Radiology and Biomedical Imaging, University of California, San Francisco, 185 Berry St, Suite 350, San Francisco, CA 94107, Jan.Neumann@tum.de.

Competing interest statement

None of the authors have any financial or other interests related to the manuscript submitted to Skeletal Radiology that might constitute a potential conflict of interest. 


\section{Keywords}

Knee osteoarthritis; diabetes mellitus; magnetic resonance imaging; risk factors; biomarkers

\section{INTRODUCTION}

With a continuously rising incidence and prevalence, osteoarthritis (OA) has become one of the leading causes of musculoskeletal disabilities in the elderly [1]. Early studies suggested that OA was only a mechanical disease due to wear and tear with joint overloading and local biomechanical insults [2]. However, more recent work demonstrated that an aberrant immunometabolism also plays a substantial key role in the pathogenesis of OA [3]. Particularly, the influence of a hyperglycemia-induced systemic inflammation has been recently under investigation of being a potential risk factor with impact on the progression of OA [4]. In hyaline cartilage, the hyperglycemic environment was shown to initiate the accumulation of advanced glycation products (AGEs) [5, 6], that lead to an increased catabolic activity of chondrocytes [7] and ultimately may accelerate cartilage degeneration in the joint of diabetics. Moreover, the possible effects of this have been shown in recent studies, furthering our understanding of a potentially underlying relationship between diabetes mellitus and OA: Exposed to the hyperglycemic environment, the articular cartilage in diabetics was shown to be more soft and permeable [8], potentially indicating reduced biomechanical properties, with a higher incidence for severe hip and knee OA [9], and ultimately an increased risk of joint arthroplasties [10]. Furthermore, a recent study using MR based quantitative compositional T2 relaxometry measurements of the knee cartilage in diabetics, showed that having diabetes was associated with an accelerated degeneration of the hyaline knee cartilage matrix composition [11]. While compositional MRI techniques are well suited to visualize early changes in the usually slow turnover and synthesis of the cartilage matrix $[12,13]$, no study has been done so far on assessing MRI structural changes in the knee joints of diabetics using semi-quantitative MRI scoring techniques. In particular assessing the impact of diabetes on changes in knee structural beyond articular cartilage such as changes in the subchondral bone, menisci, and ligamentous structures would be of interest. We hypothesize that diabetics demonstrate faster progression of knee structural abnormalities compared with diabetes-free controls over a 4 -year follow-up period. Therefore, the purpose of our study was to longitudinally investigate osteoarthritis related knee structural changes over 4 years in diabetics, using semi-quantitative MRI scoring, and to determine if diabetics show accelerated tissue degeneration when compared to diabetesfree controls.

\section{MATERIAL \& METHODS}

\section{The Osteoarthritis Initiative Database}

All participants in this study were selected from the Osteoarthritis Initiative (OAI) database, a multicenter cohort study of knee osteoarthritis, sponsored by the U.S. National Institutes of Health (NIH) for investigating diagnosis, treatment, and prevention of OA. Starting in February 2004 to May 2006 and completed as of January 1st 2015, the longitudinal clinical and imaging data of 4796 participants were collected over a nine-year period at four clinical 
centers (University of Maryland, Baltimore, Maryland; Memorial Hospital of Rhode Island / Brown University, Pawtucket, Rhode Island; Ohio State University, Columbus, Ohio; University of Pittsburgh, Pittsburgh, Pennsylvania). The entire cohort consists of an ethnically diverse group of women and men aged $45-79$ years who have knee OA, are at risk for developing knee OA or have no known risk factors for OA. Overall, the aim of the OAI was to establish a public-domain research resource and to investigate the scientific evaluation of biomarkers for $\mathrm{OA}$ as potential surrogate endpoints for disease onset and its progression (https://oai.epi-ucsf.org/).

\section{Selection of participants from the OAI}

Participants were eligible for inclusion if they had no or early stage knee OA (grade $0-2$; Kellgren-Lawrence (K/L) classification of osteoarthritis) in the right knee at baseline, whereas, participants with moderate to end-stage (K/L 3-4 or knee replacement) OA in the right knee were excluded. Reasons for the exclusion of participants with moderate to endstage OA were to maintain a study cohort with allegedly sufficient knee structure abnormalities that longitudinally potentially worsen when exposed to a osteoarthritic risk factor. Furthermore, we did choose the right knee as this is typically the dominant knee and potentially at higher risk for degeneration and, moreover, as previously shown, focal lesion of the knee of OAI participants were highly correlated between the right and left knee [14]. All patients had to have complete baseline data on sex, BMI, and age as well as complete right knee MRI scans available at baseline and the 48 month visit. Exclusion criteria included a medical history of rheumatoid arthritis or another inflammatory arthropathy. In the next step, for all participants that fulfilled the criteria above, the medical history from all visits within the first 4 years (Baseline, 12-month, 24-month, 36-month, and 48-month visits) was then reviewed to identify participants with diabetes based on a self-administered questionnaire that asked participants if a doctor told them they had diabetes or high blood sugar [15] and the use of insulins and antidiabetic agents reported on the prescription medication inventory. In total, 244 participants with diabetes fulfilled the selection criteria. Secondly, a diabetes-free control group of equal size ( 244 controls) was selected and matched in small strata based on age, sex, BMI, and K/L score with the diabetic group. The strata were defined as follows: sex (male or female), K/L score (0-1 or 2), age (45-49, 50$54,55-59,60-64,65-69,70-74,75-79)$ and BMI $\left(2.5 \mathrm{~kg} / \mathrm{m}^{2}\right.$ intervals from 17.5 to $\left.>45.0\right)$. Figure 1 shows a flow chart illustrating patient selection for this study.

\section{Participants characteristics and medical history}

For all participants that fulfilled the selection criteria, Physical Activity Scale for the Elderly (PASE) data as well as history of knee injury (knee ever injured so badly difficult to walk for at least one week) and knee surgery (incl. arthroscopy, ligament repair, meniscectomy) were collected.

\section{Severe diabetes and diabetes related complications}

Participants with self-reported diabetes were categorized as having a severe diabetic course if either diabetes-related organ complications (diabetic retinopathy or diabetic nephropathy) and/or treatment with insulin injections were reported [15]. Diabetics with no reported complication and no insulin medication on medical record were categorized as diabetics 
without severe disease. In total, ninety-nine participants from the diabetes group reported complications, of which 9 (9.1\%) reported diabetic nephropathy, $45(45.5 \%)$ reported diabetic retinopathy and $14(14.1 \%)$ reported both, nephropathy and retinopathy, complications. Furthermore, fifty-eight percent (58.6\%) reported treatment with insulin.

\section{MRI protocol and Image Analysis}

All scans were acquired using 3.0T MRI systems (Siemens Magnetom Trio; Siemens Healthcare, Erlangen, Germany) with quadrature transmit-receive coils (USA Instruments, Aurora, OH, USA) at the four different OAI clinical sites. For the morphological MRI analysis of the knee, using the Whole-Organ Resonance Imaging Score (WORMS), the following sequences from the MR imaging protocol were used: a 3D dual echo steady-state (DESS) gradient-echo with water excitation (WE) sequence obtained in the sagittal plane (16.3/4.7/25 $5^{\circ}$ TR/TE/flip angle), a 3D T1-weighted fast low-angle shot (FLASH) gradientecho with WE sequence in a coronal plane (20/7.57/12 ${ }^{\circ}$, TR/TE/flip angle) and two 2D proton density/intermediate-weighted sequences in a coronal plane $(3700 \mathrm{~ms} / 29 \mathrm{~ms}$, TR/TE) and in a sagittal plane $(3200 \mathrm{~ms} / 30 \mathrm{~ms}$, TR/TE, with fat suppression (FS)).

All readings were performed using the modified WORMS scoring [16] independently by two radiologists (JN and JBG) with 7 years of experience in musculoskeletal imaging, both radiologists were blinded to the clinical information and the diabetes status. The modified WORMS grading has been described in detail in earlier studies [17-19]. Briefly, all incorporated features in the image analysis (articular cartilage, bone marrow patterns, subarticular cysts, ligaments, effusion, loose bodies, and popliteal cysts) were assessed on a numeric scoring system, grading the severity of morphological findings in ascending order. Cartilage integrity was assessed based on the extent of the joint cartilage surface, area, depth or volume involved (graded $0-6$ ). Similarly using size and extent, bone marrow edema pattern and subarticular cysts were graded $0-3$. Structural lesions of the anterior and poster crucial ligament (ACL, PCL), medial and lateral collateral ligament (MCL, LCL), the patellar tendon and the popliteal tendon were graded from 0 to 4 . Lesions of the lateral and medial meniscus were further subdivided into anterior, posterior horn or body and scored separately based from 0 to 4 . Finally, joint effusion, loose bodies, and popliteal cysts were all graded from $0-3$.

\section{Statistical Analysis}

Statistical analysis was performed using STATA software (Version 14, College Station, TX: StataCorp LP) with $\mathrm{p}<0.05$ being considered as statistically significant. Demographic data between the study cohorts were assessed using either Pearson's $\chi 2$-test or independent t-test as appropriate. Linear regression models were used to assess the differences between diabetics and diabetes-free controls in the change over time of the modified WORMS features. All analyses were adjusted for the common risk factors of knee OA (age, sex, $\mathrm{BMI}$ ), and $\mathrm{K} / \mathrm{L}$ score. The participant demographics in our study groups revealed a significantly higher percentage of African American in the diabetes group. Due to important racial differences in joint tissue markers [20] we also adjusted for race. 
For the inter- and intrareader reliability the intraclass correlation (ICC) estimates and their 95\% confidence intervals were calculated based on a mean-rating $(k=2)$, absolute agreement, 2-way mixed-effects model, where ICC values less than 0.5 indicated poor reliability, values between 0.5 and 0.75 indicated moderate reliability, values between 0.75 and 0.9 indicated good reliability, and values greater than 0.90 indicated excellent reliability [21].

\section{RESULTS}

\section{Participant demographics}

As shown in Table 1, both the diabetes and diabetes-free control group were well matched, with no significant differences for age, sex, BMI, and $\mathrm{K} / \mathrm{L}$ score $(\mathrm{p}>0.20)$. With respect to the racial distribution, the diabetic group showed a significantly higher percentage of African American when compared to the diabetes-free controls $(\mathrm{p}<0.001)$. With respect to the physical activity scores (PASE), the diabetic group was found to have minimally lower PASE activity levels relative to controls that, however, did not translate into a statistical difference ( $\mathrm{p}=0.26$ ). The history of knee injury as well as the history of knee surgery showed no significant difference between the two groups.

\section{Cross-sectional analysis of WORMS findings in diabetics and diabetes-free controls at study enrollment}

At the time of enrollment, the two age, sex, BMI, and K/L score matched groups did not show significant differences with respect to morphological abnormalities as measured by WORMS sum and subscores. The overall WORMS score was higher in participants with diabetes, as were the global cartilage and meniscus WORMS scores but none of the differences showed statistical significance. Table 2 shows the morphological differences between diabetics and diabetes-free controls at the time point of enrollment.

\section{Longitudinal, structural changes in diabetics and diabetes-free controls over $\mathbf{4 8}$ months}

Table 3 shows the longitudinal change in morphological knee abnormalities by group. Participants in the diabetes group showed a significantly higher increase in the overall WORMS score when compared to diabetes-free controls (increase in WORMS [95\% CI]: $4.87[4.17,5.57]$ vs 3.23 [2.60, 3.85], $\mathrm{p}=0.001$ for comparing changes).

The results were consistent with the findings of the cartilage lesion subscores. The mean increase in the WORMS cartilage subscores was more pronounced in diabetics not only for the global WORMS cartilage knee score but also for every single compartment cartilage score (Figure 2 and 3). Moreover, changes were significantly greater for the global WORMS cartilage score $(\mathrm{p}<0.001)$, patella $(\mathrm{p}=0.005)$, lateral tibia $(\mathrm{p}=0.03)$, and both femoral compartments (lowest $\mathrm{p}$-value; $\mathrm{p}=0.001$ ).

When looking at compartments separately, the differences between the two groups were most prominent in the patella $(0.60[0.48,0.73]$ diabetics vs $0.35[0.24,0.45]$ diabetes-free controls, $\mathrm{p}=0.005$ ) and both femoral compartments (lateral: $0.43[0.30,0.55]$ vs $0.18[0.11$, $0.25], \mathrm{p}=0.001$; medial: 0.45 [0.33, 0.57] vs 0.24 [0.13, 0.34], $\mathrm{p}=0.01)$. 
A significantly greater increase was also found for the diabetics with respect to the WORMS meniscus subscores (Figure 4). For changes in the meniscus subscores, a significantly higher increase for WORMS meniscus subscores was observed for diabetics in the global WORMS meniscus score $(\mathrm{p}=0.001)$ but also separately for the medial $(\mathrm{p}=0.01)$ and lateral $(\mathrm{p}=0.01)$ meniscus.

Changes in the adjacent bone, including bone marrow edema pattern and subarticular cysts, as well as changes for ligamentous lesions, effusion and popliteal cysts were not significantly different between the two groups.

\section{Longitudinal, structural changes in severe diabetics and diabetes-free controls over 48 months}

Similar to the findings in Table 3, the subanalysis of diabetics with severe disease showed a significantly greater increase in the overall WORMS score when compared to diabetes-free controls. The differences were even greater than in the overall diabetes cohort (increase in WORMS [95\% CI]: $5.83[4.52,7.14]$ vs $3.21[2.60,3.82]$, $\mathrm{p}<0.001)$.

Diabetics with severe disease showed a significantly greater increase in the global WORMS cartilage score $(\mathrm{p}<0.001)$ when compared to diabetes-free controls. Furthermore, a higher increase was again seen in all separate cartilage compartments with significantly higher values in the patella $(\mathrm{p}=0.01)$, medial femur $(\mathrm{p}=0.004)$, and lateral femur $(\mathrm{p}=0.002)$. Among the different compartments, the lateral femur in diabetics with severe disease showed the highest difference compared with the diabetes-free control group $(0.61[0.35,0.87]$ vs 0.18 $[0.10,0.25], \mathrm{p}=0.002)$.

Additionally, diabetics with severe disease showed a significantly greater increase for the global WORMS meniscus score $(\mathrm{p}=0.004)$ as well as lateral meniscus $(\mathrm{p}=0.01)$ relative to diabetes-free controls. Changes in the medial meniscus were higher as well but did not reach statistical significance $(\mathrm{p}=0.06)$. Changes for bone marrow edema pattern, subarticular cysts, ligaments, effusion and popliteal cysts were not significantly different between the two groups.

When comparing diabetics with severe disease to diabetics with non-severe disease (Table 4) the latter showed on average a slower mean rate of change but the differences were overall not significant.

\section{Longitudinal, structural changes in non-severe diabetics and diabetes-free controls over 48 months}

Comparing participants with non-severe diabetes and diabetes-free controls, the longitudinal increase of overall WORMS score was significantly higher for the non-severe diabetes (4.39 $[3.53,5.25]$ vs $3.24[2.63,3.86], \mathrm{p}=0.03)$. Similar to that, the increase was also greater for all separate cartilage compartments with significantly higher values for the patella $(\mathrm{p}=0.03)$, lateral femur $(\mathrm{p}=0.002)$ as well as for the global WORMS cartilage score $(\mathrm{p}=0.002)$. A significantly greater increase for the global WORMS meniscus score $(\mathrm{p}=0.02)$ was also observed for non-severe diabetics when compared to diabetes-free controls. The separate analysis of the medial and lateral meniscus compartment did also show a higher increase but 
did not translate into statistical significance ( $\mathrm{p}=0.05$ and $\mathrm{p}=0.13$, respectively). In accordance with the other group comparisons, changes for bone marrow edema pattern and subarticular cysts were not significantly different between non-severe diabetics and diabetes-free controls. Table 5 shows the longitudinal changes of WORMS findings in non-severediabetics and diabetes-free controls over 48 months.

\section{Inter-/Intrareader Reproducibility for WORMS gradings}

The ICCs for interobserver agreement were 0.97 (95\% CI: 0.95, 0.98) for WORMS cartilage, 0.97 (95\% CI: 0.95, 0.98) for WORMS menisci, 0.95 (95\% CI: 0.92, 0.97) for WORMS ligaments, 0.82 (95\% CI: 0.71, 0.88) for WORMS bone marrow edema pattern, 0.96 (95\% CI: $0.94,0.97)$ for WORMS subarticular cyst, and 0.75 (95\% CI: $0.52,0.86$ ) for WORMS effusion, loose bodies and popliteal cysts.

The ICCs for intraobserver agreement were 0.99 (95\% CI: 0.98, 0.99) and 0.99 (95\% CI: 0.99, JBG 0.99) for WORMS cartilage, 0.96 (95\% CI: 0.94, 0.97) and 0.96 (95\% CI: 0.94, 0.97 ) for WORMS menisci, 0.95 (95\% CI: 0.92, 0.97) and 0.96 (95\% CI: 0.94, 0.97) for WORMS ligaments, 0.98 (95\% CI: 0.97, 0.98) and 0.98 (95\% CI: 0.97, 0.98) for WORMS bone marrow edema pattern, 0.98 (95\% CI: $0.97,0.99)$ and 0.85 (95\% CI: $0.72,0.91)$ for WORMS subarticular cyst, and 0.90 (95\% CI: $0.81,0.94)$ and 0.74 (95\% CI: $0.52,0.86$ ) for WORMS effusion, loose bodies and popliteal cysts.

\section{DISCUSSION}

Our study investigated cross-sectional and longitudinal changes in knee structural abnormalities in diabetics using semi-quantitative knee MRI scoring and compared them to diabetes-free controls. We found that within 4 years of follow-up, diabetics exhibited a significantly greater increase in cartilage and meniscus lesions. In analyses stratified by diabetes severity, we found an even greater deterioration of knee structures in diabetics with more severe diabetes as documented by treatment with insulin and presence of diabetic renal and ophthalmological complications. The results of our study show that diabetics exhibit a stronger deterioration of knee structure and are therefore potentially at higher risk for developing knee OA.

$\mathrm{OA}$ and diabetes are both common and costly chronic diseases, presenting a challenge for today's health care system and interfere with the quality of life of patients suffering from these diseases [22]. Recent studies have suggested a potential underlying relationship of these diseases $[6,7,9,23,24]$ and indicated that diabetes may be a risk factor for the development of OA. Based on epidemiological and experimental data, Berenbaum proposed that diabetes might be an independent risk factor for osteoarthritis and, moreover, that the identification of diabetes as a risk factor would have substantial impact on prevention of OA initiation and progression [4]. This statement is strengthened by the fact that there is still no known cure and no proven pharmacologic intervention available to the general population to treat OA $[25,26]$ and therefore the identification of potential risk factors would be the closest step to slow down or even prevent the onset of OA. 
Targeting obesity as a long time known contributor to the risk profile of OA patients, it has been recently demonstrated how the identification and optimization of risk factors can be a tipping point for the treatment and prevention of OA. It has been clearly demonstrated that obesity is associated with a higher prevalence and severity of knee OA but also with progression of cartilage lesions [27]. In this context by focusing on weight loss in obese and overweight subjects, Gersing et al. were able to show that weight loss significantly decreased progression of cartilage structural and compositional degeneration $[17,28]$. These studies show well how the usually irreversible progress of joint destruction could be potentially prevented by identifying patients that are at risk, addressing the risk factors and ultimately improving the quality of life of individuals who are affected by this disease. Therefore, a further understanding of how other risk factors, such as diabetes, potentially may be involved in the degenerative process of OA will be of substantial help to understand $\mathrm{OA}$ and, moreover, contribute to developing effective treatment and preventive strategies.

To date, several studies have already advanced our understanding of the relationship between OA and diabetes $[6,7,9,23,24]$ but none of these studies investigated the longitudinal change of structural abnormalities using MRI. While some studies were able to show that diabetes is associated with a higher likelihood of receiving joint arthroplasties [9] and also is a predictor of joint space reduction [23], other studies pointed out an altered cartilage composition, leading to possibly decreased biomechanical stability [7, 24, 29]. In the latter studies, the focus was set on the overproduction and accumulation of AGEs [30]. Triggered by an hyperglycemic environment, AGE-associated altered tensile properties of the hyaline cartilage may result in an increased cartilage stiffness and fragility [29]; thus ultimately the cartilage may become increasingly prone to damage and degeneration. The results in our current study support this observation. When compared to diabetes-free controls, we found diabetics to exhibit a higher rate of degradation. Using MRI based semi-quantitative scoring of structural knee abnormalities our results showed that diabetics have a significantly higher increase in cartilage lesions over 4 years when compared to diabetes-free controls. These differences were also demonstrated in our subanalysis of severe and non-severe diabetics. Both diabetes groups separately showed worse WORMS scores when compared to the diabetes-free control group, whereas, the differences were overall more pronounced in the diabetics with severe disease compared to the diabetes-free controls, indicating that the severity of the diabetic disease correlates with an accelerated deterioration of the cartilage. Additionally, severe-diabetics showed also a higher increase in WORMS lesions when compared to diabetics without severe disease. However, these results did not show statistically difference, indicating that the main difference of our results is not driven by severe diabetes alone.

In addition to the greater increase in cartilage lesions in diabetic participants, our results also revealed a greater increase in meniscus lesions which were again most prominent in severe diabetics. To the best of our knowledge, these results have not been shown in previous studies. A previous study investigating the potential effect of insulin-like growth factor-I (IGF-I) in promoting regeneration of meniscal tissue, Tumia et al. were able to show that fibrochondrocytes in meniscal tissue have the ability to regenerate when exposed to IGF-I [31], however, in patients with diabetes the physiological growth hormone (GH) and Insulinlike growth factor (IGF) axis are disrupted, resulting in decreased IGF-1 levels [32] in 
patients younger than 65 years. Therefore, the decreased bioavailability of IGF-1 in diabetics may lead to an impaired regeneration of the meniscal tissue and ultimately making diabetics more susceptible to developing meniscal lesions. Although this potentially explains our findings of increased temporal alterations in menisci of diabetics, the deterioration of the cartilage and menisci could also result from a direct impact of these two joint structures onto each other: meniscal damage could occur along with the cartilage breakdown due to mechanical tearing or vice versa. Both abnormalities have been shown to substantially contribute to narrowing of joint space [33]. However, to which extent both abnormalities affect each other still remains to be determined.

Interestingly, changes in the adjacent bone, such as bone marrow edema pattern and subarticular cysts, were not significantly different between diabetics and diabetes-free controls in our study. For bone marrow lesions, a positive correlation with the severe and accelerated cartilage degeneration is generally expected $[34,35]$ and the presence of bone marrow lesions also strongly predict the incidence of subarticular cysts [36]. Since studies suggest that bone marrow lesions are most probably reflecting increased tissue vascularity accompanying increased remodeling activity [37] our results may be explained by the fact, that diabetics present with a vascular rarefaction which ultimately also affects the bone marrow remodeling through a shortage of vascular regenerative cells [38] and therefore possibly antagonizes the expected changes in the adjacent bone when cartilage damage occurs.

We acknowledge that this study has several limitations. Firstly, the definition of severity in our study is based on self-reported diabetes related complications and the medication history. No information on diabetes duration and on laboratory markers which measure longterm blood glucose levels (HbA1c) is available in the OAI. This information would have been helpful to assess the quality of each participant's diabetes management. Secondly, the diabetics in our study cohort were on average obese with a BMI of greater than $30 \mathrm{~kg} / \mathrm{m}^{2}$. When studying the effects of diabetes in an overweight cohort it is important to look at the effects of diabetes independently from BMI. In order this, we not only matched our groups for BMI but also and adjusted our analysis for BMI. Thirdly, no information on the age at diagnosis of diabetes was available in the OAI database. However, we ensured that all of our participants with diabetes maintained their diabetes for the complete study as well as that the diabetes-free controls were free of self-reported diabetes over the entire follow-up period. Finally, no information on the subtype of diabetes (type 1 or 2) is available in the OAI. However, due to the average age of all our participants of being older than 63 years, it is expected that the majority of patients had type 2 diabetes since this diabetes subtype accounts for about $90 \%$ of all diagnosed cases of diabetes mellitus in this age range [39].

In conclusion, we were able to show that when compared to diabetes-free controls, diabetics showed a significantly higher increase of structural abnormalities with respect to cartilage and meniscus lesions over a time period of 4 years. Both effects were even more prominent in diabetics with severe diabetes disease. Our results suggest, that diabetics are at higher risk of developing OA through accelerated cartilaginous and meniscal breakdown. 


\section{Acknowledgements}

We would like to thank the participants and staff of the Coordinating Center of the OAI for their invaluable assistance with patient selection, statistical analysis, and technical support.

Role of Funding Source:

The analyses in this study were funded through the NIH/NIAMS (National Institute of Arthritis and Musculoskeletal and Skin Diseases grants R01AR064771 and P50-AR060752). The study was also supported by the Osteoarthritis Initiative, a public-private partnership comprising $5 \mathrm{NIH}$ contracts (National Institute of Arthritis and Musculoskeletal and Skin Diseases contracts N01-AR-2-2258, N01-AR-2-2259, N01-AR-2-2260, N01-AR-22261, and N01-AR-2-2262), with research conducted by the Osteoarthritis Initiative Study Investigators. The study was also funded in part by the Intramural Research Program of the National Institute on Aging, NIH. Private funding partners include Merck Research, Novartis Pharmaceuticals, GlaxoSmithKline, and Pfizer; the private sector funding for the Novartis Pharmaceuticals, GlaxoSmithKline, and Pfizer; the private sector funding for the Osteoarthritis Initiative is managed by the Foundation for the National Institutes of Health.

\section{REFERENCES}

1. United States Bone and Joint Initiative: The Burden of Musculoskeletal Diseases in the United States (BMUS), Third Edition, 2014 Rosemont, IL Available at http://www.boneandjointburden.org. Accessed on May 5, 2018.

2. Felson DT. Osteoarthritis as a disease of mechanics. Osteoarthr Cartil OARS Osteoarthr Res Soc 2013;21:10-15. 10.1016/j.joca.2012.09.012

3. Mobasheri A, Rayman MP, Gualillo O, Sellam J, van der Kraan P, Fearon U. The role of metabolism in the pathogenesis of osteoarthritis. Nat Rev Rheumatol 2017;13:302-311. 10.1038/nrrheum. 2017.50 [PubMed: 28381830]

4. Berenbaum F Diabetes-induced osteoarthritis: from a new paradigm to a new phenotype. Postgrad Med J 2012;88:240-242. 10.1136/pgmj.2010.146399rep [PubMed: 22441236]

5. Degenhardt TP, Thorpe SR, Baynes JW. Chemical modification of proteins by methylglyoxal. Cell Mol Biol Noisy--Gd Fr 1998;44:1139-1145

6. King KB, Rosenthal AK. The adverse effects of diabetes on osteoarthritis: update on clinical evidence and molecular mechanisms. Osteoarthritis Cartilage 2015;23:841-850. 10.1016/j.joca. 2015.03.031 [PubMed: 25837996]

7. Steenvoorden MMC, Huizinga TWJ, Verzijl N, et al. Activation of receptor for advanced glycation end products in osteoarthritis leads to increased stimulation of chondrocytes and synoviocytes. Arthritis Rheum 2006;54:253-263. 10.1002/art.21523 [PubMed: 16385542]

8. Athanasiou KA, Fleischli JG, Bosma J, et al. Effects of diabetes mellitus on the biomechanical properties of human ankle cartilage. Clin Orthop Relat Res 1999;182-9

9. Schett G, Kleyer A, Perricone C, et al. Diabetes is an independent predictor for severe osteoarthritis: results from a longitudinal cohort study. Diabetes Care 2013;36:403-409. 10.2337/dc12-0924 [PubMed: 23002084]

10. King KB, Findley TW, Williams AE, Bucknell AL. Veterans With Diabetes Receive Arthroplasty More Frequently and at a Younger Age. Clin Orthop 2013;471:3049-3054. 10.1007/ s11999-013-3026-3 [PubMed: 23649224]

11. Neumann J, Hofmann FC, Heilmeier U, et al. Type 2 diabetes patients have accelerated cartilage matrix degeneration compared to diabetes free controls: data from the Osteoarthritis Initiative. Osteoarthritis Cartilage 2018;26:751-761. 10.1016/j.joca.2018.03.010 [PubMed: 29605381]

12. Sophia Fox AJ, Bedi A, Rodeo SA. The basic science of articular cartilage: structure, composition, and function. Sports Health 2009;1:461-8. 10.1177/1941738109350438 [PubMed: 23015907]

13. Liebl H, Joseph G, Nevitt MC, et al. Early T2 changes predict onset of radiographic knee osteoarthritis: data from the osteoarthritis initiative. Ann Rheum Dis 2015;74:1353-9. 10.1136/ annrheumdis-2013-204157 [PubMed: 24615539]

14. Chundru R, Baum T, Nardo L, et al. Focal knee lesions in knee pairs of asymptomatic and symptomatic subjects with OA risk factors--data from the Osteoarthritis Initiative. Eur J Radiol 2013;82:e367-373. 10.1016/j.ejrad.2013.02.038 [PubMed: 23540944] 
15. Charlson ME, Pompei P, Ales KL, MacKenzie CR. A new method of classifying prognostic comorbidity in longitudinal studies: development and validation. J Chronic Dis 1987;40:373-383 [PubMed: 3558716]

16. Peterfy CG, Guermazi A, Zaim S, et al. Whole-Organ Magnetic Resonance Imaging Score (WORMS) of the knee in osteoarthritis. Osteoarthritis Cartilage 2004;12:177-90. 10.1016/j.joca. 2003.11.003 [PubMed: 14972335]

17. Gersing AS, Schwaiger BJ, Nevitt MC, et al. Is Weight Loss Associated with Less Progression of Changes in Knee Articular Cartilage among Obese and Overweight Patients as Assessed with MR Imaging over 48 Months? Data from the Osteoarthritis Initiative. Radiology 2017;161005 10.1148/ radiol.2017161005

18. Alizai H, Virayavanich W, Joseph GB, et al. Cartilage lesion score: comparison of a quantitative assessment score with established semiquantitative MR scoring systems. Radiology 2014;271:479-487. 10.1148/radiol.13122056 [PubMed: 24475848]

19. Joseph GB, McCulloch CE, Nevitt MC, et al. Tool for osteoarthritis risk prediction (TOARP) over 8 years using baseline clinical data, X-ray, and MRI: Data from the osteoarthritis initiative. J Magn Reson Imaging JMRI 2017 10.1002/jmri.25892

20. Jordan JM. An Ongoing Assessment of Osteoarthritis in African Americans and Caucasians in North Carolina: The Johnston County Osteoarthritis Project. Trans Am Clin Climatol Assoc 2015;126:77-86 [PubMed: 26330661]

21. Portney Leslie G. W Mary P. Foundations of Clinical Research: Applications to Practice, 3rd ed. 2009 Prentice Hall, Upper Saddle River, NJ

22. Turkiewicz A, Petersson IF, Björk J, et al. Current and future impact of osteoarthritis on health care: a population-based study with projections to year 2032. Osteoarthritis Cartilage 2014;22:1826-1832. 10.1016/j.joca.2014.07.015 [PubMed: 25084132]

23. Eymard F, Parsons C, Edwards MH, et al. Diabetes is a risk factor for knee osteoarthritis progression. Osteoarthritis Cartilage 2015;23:851-859. 10.1016/j.joca.2015.01.013 [PubMed: 25655678]

24. Rosa SC, Rufino AT, Judas FM, Tenreiro CM, Lopes MC, Mendes AF. Role of glucose as a modulator of anabolic and catabolic gene expression in normal and osteoarthritic human chondrocytes. J Cell Biochem 2011;112:2813-2824. 10.1002/jcb.23196 [PubMed: 21608018]

25. Yusuf E Pharmacologic and Non-Pharmacologic Treatment of Osteoarthritis. Curr Treat Options Rheumatol 2016;2:111-125. 10.1007/s40674-016-0042-y

26. Karsdal MA, Michaelis M, Ladel C, et al. Disease-modifying treatments for osteoarthritis (DMOADs) of the knee and hip: lessons learned from failures and opportunities for the future. Osteoarthritis Cartilage 2016;24:2013-2021. 10.1016/j.joca.2016.07.017 [PubMed: 27492463]

27. Laberge MA, Baum T, Virayavanich W, et al. Obesity increases the prevalence and severity of focal knee abnormalities diagnosed using 3T MRI in middle-aged subjects--data from the Osteoarthritis Initiative. Skeletal Radiol 2012;41:633-641. 10.1007/s00256-011-1259-3 [PubMed: 21887596]

28. Gersing AS, Solka M, Joseph GB, et al. Progression of cartilage degeneration and clinical symptoms in obese and overweight individuals is dependent on the amount of weight loss: 48month data from the Osteoarthritis Initiative. Osteoarthritis Cartilage 2016;24:1126-1134. 10.1016/j.joca.2016.01.984 [PubMed: 26828356]

29. Verzij1 N, DeGroot J, Ben ZC, et al. Crosslinking by advanced glycation end products increases the stiffness of the collagen network in human articular cartilage: a possible mechanism through which age is a risk factor for osteoarthritis. Arthritis Rheum 2002;46:114-123.

10.1002/1529-0131(200201)46:1\&1t;114::AID-ART10025\&gt;3.0.CO;2-P [PubMed: 11822407]

30. Singh VP, Bali A, Singh N, Jaggi AS. Advanced glycation end products and diabetic complications. Korean J Physiol Pharmacol Off J Korean Physiol Soc Korean Soc Pharmacol 2014;18:1-14. 10.4196/kjpp.2014.18.1.1

31. Tumia NS, Johnstone AJ. Regional regenerative potential of meniscal cartilage exposed to recombinant insulin-like growth factor-I in vitro. J Bone Joint Surg Br 2004;86:1077-1081 [PubMed: 15446543]

32. Teppala S, Shankar A. Association Between Serum IGF-1 and Diabetes Among U.S. Adults. Diabetes Care 2010;33:2257-2259. 10.2337/dc10-0770 [PubMed: 20639451] 
33. Hunter DJ, Zhang YQ, Tu X, et al. Change in joint space width: hyaline articular cartilage loss or alteration in meniscus? Arthritis Rheum 2006;54:2488-2495. 10.1002/art.22016 [PubMed: $16868968]$

34. Zhao J, Li X, Bolbos RI, Link TM, Majumdar S. Longitudinal assessment of bone marrow edemalike lesions and cartilage degeneration in osteoarthritis using $3 \mathrm{~T}$ MR T1rho quantification. Skeletal Radiol 2010;39:523-531. 10.1007/s00256-010-0892-6 [PubMed: 20195865]

35. Maas O, Joseph GB, Sommer G, Wild D, Kretzschmar M. Association between cartilage degeneration and subchondral bone remodeling in patients with knee osteoarthritis comparing MRI and (99m)Tc-DPD-SPECT/CT. Osteoarthritis Cartilage 2015;23:1713-1720. 10.1016/j.joca. 2015.05.014 [PubMed: 26028141]

36. Crema MD, Roemer FW, Zhu Y, et al. Subchondral cystlike lesions develop longitudinally in areas of bone marrow edema-like lesions in patients with or at risk for knee osteoarthritis: detection with MR imaging--the MOST study. Radiology 2010;256:855-862. 10.1148/radiol.10091467 [PubMed: 20530753]

37. Shabestari M, Vik J, Reseland JE, Eriksen EF. Bone marrow lesions in hip osteoarthritis are characterized by increased bone turnover and enhanced angiogenesis. Osteoarthritis Cartilage 2016;24:1745-1752. 10.1016/j.joca.2016.05.009 [PubMed: 27233775]

38. Spinetti G, Cordella D, Fortunato O, et al. Global remodeling of the vascular stem cell niche in bone marrow of diabetic patients: implication of the microRNA-155/FOXO3a signaling pathway. Circ Res 2013;112:510-522. 10.1161/CIRCRESAHA.112.300598 [PubMed: 23250986]

39. National Diabetes Statistics Report | Data \& Statistics | Diabetes | CDC. https://www.cdc.gov/ diabetes/data/statistics/statistics-report.html. Accessed 23 May 2018 


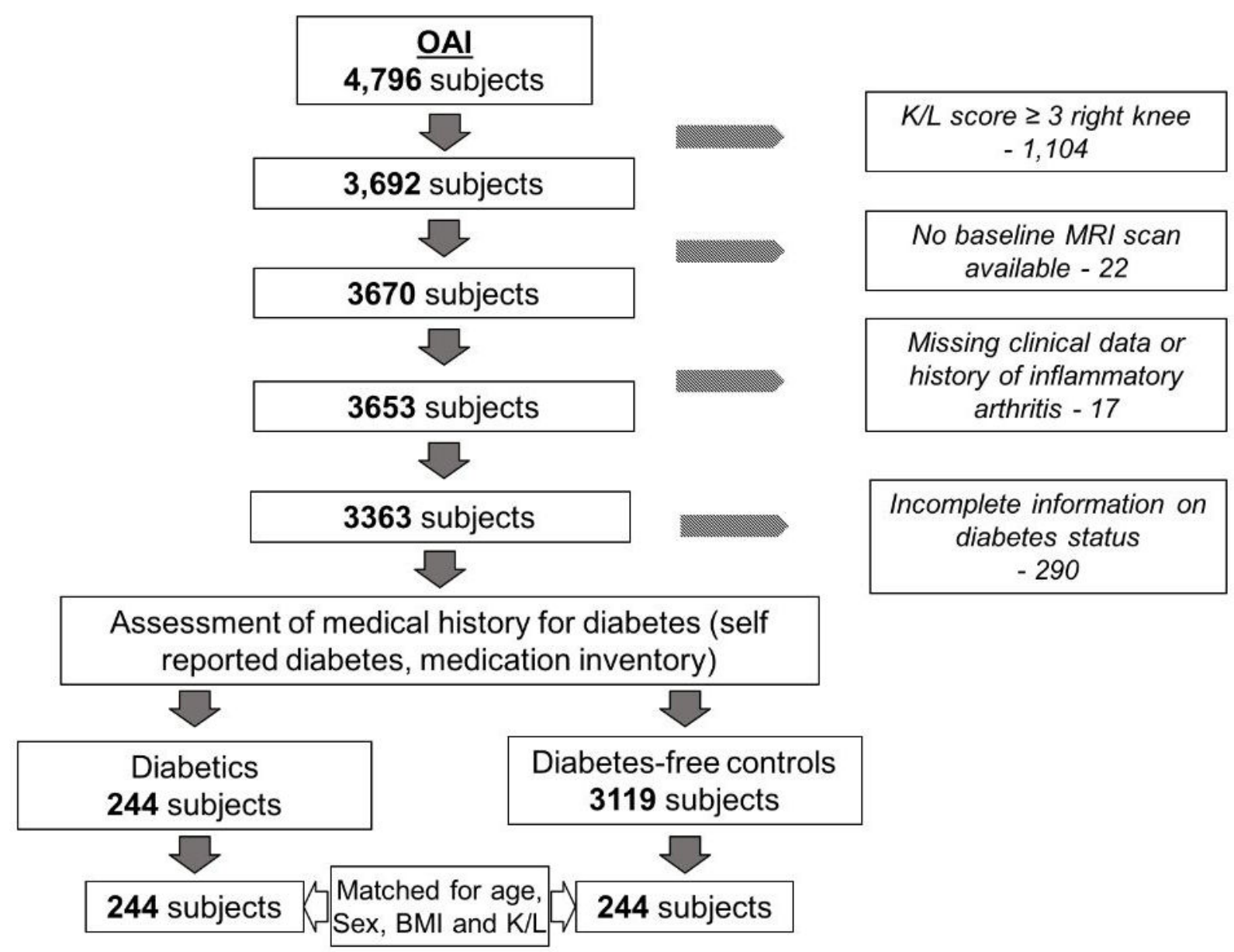

Fig 1.

Selection of study participants. Flow-chart illustrating patient selection for the present study from the overall OAI cohort. (BMI = body mass index, $\mathrm{K} / \mathrm{L}=$ radiographic Kellgren/ Lawrence score). 

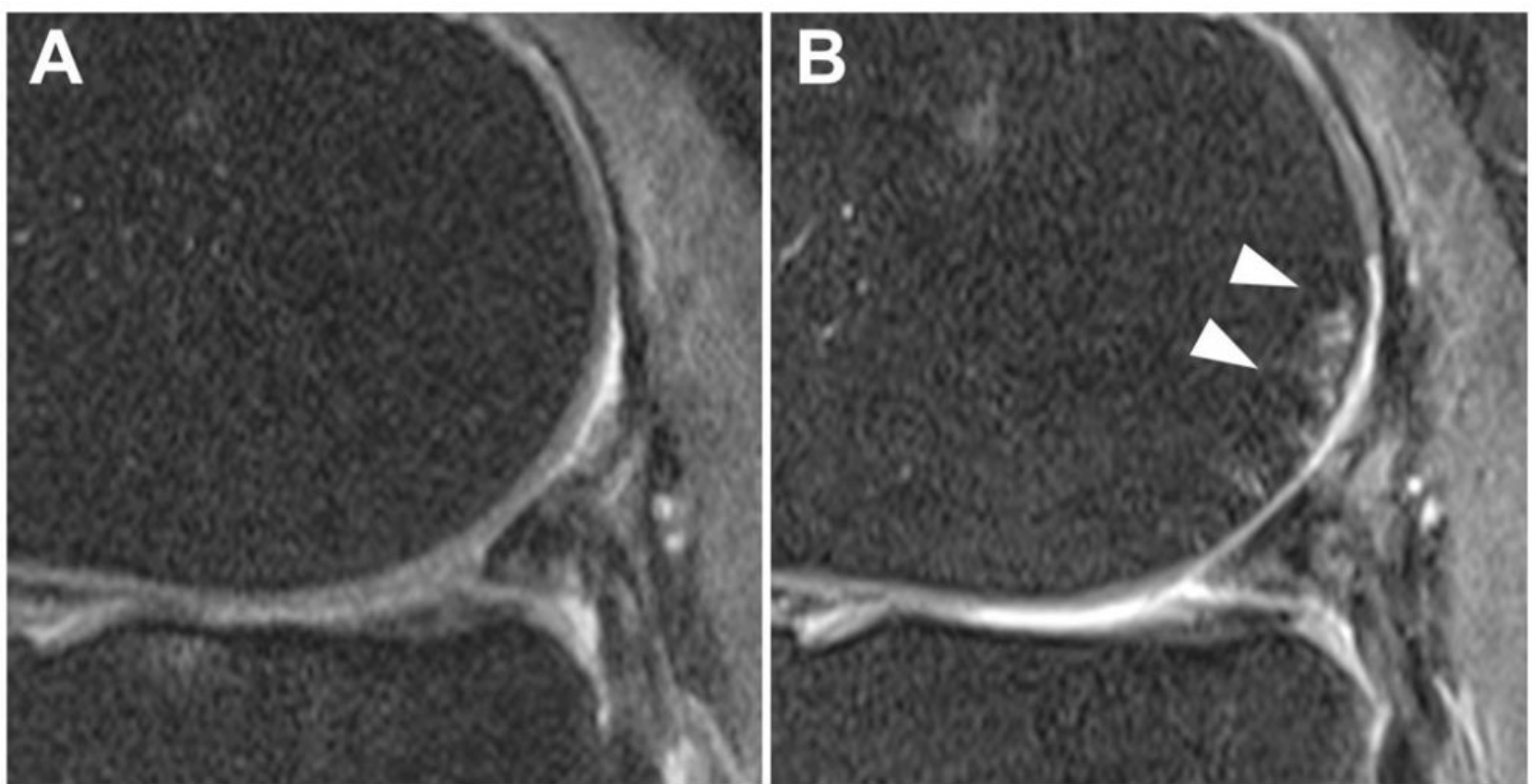

Fig 2.

Right knee of a 67-year old woman with diabetes. An intermediate-weighted (IW) sequence in the sagittal plane with fat suppression of the posterior aspect of the lateral femur at baseline (A) and at 48-month follow-up are shown (B). The posterior aspect of the lateral femoral condyle at baseline shows no visible cartilage defect, whereas, the follow-up MRI shows a newly developed large full-thickness defect (white arrowheads). Additionally, small cystic lesions and bone marrow lesions are visible in the subchondral bone right underneath the cartilage defect. There is also increased joint fluid. Also note pre-existing meniscal degenerative changes and thinning of the tibial cartilage. 

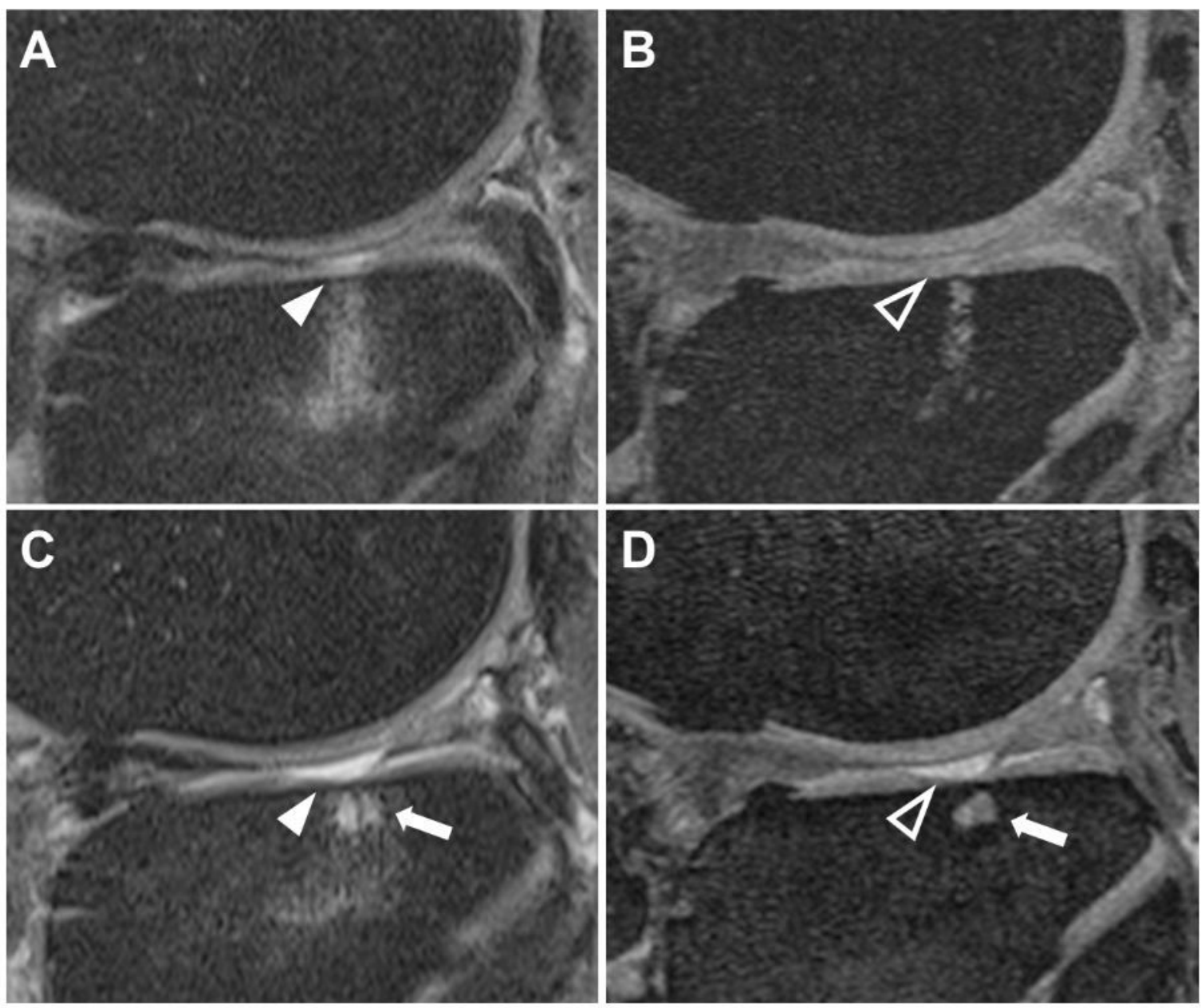

Fig 3.

Baseline and follow-up knee MRI showing a sagittal IW (A, C) and DESS sequence (B, D) with fat suppression, of a 73 year male diabetic. At baseline, a partial-thickness defect in the weight bearing area of the lateral tibia is shown, accompanied by a large bone marrow lesion in the lateral tibia (A, white arrowhead; B hollow white arrowhead). In the follow-up images at 48-month, the defect has progressed into a full-thickness defect $(\mathrm{C}+\mathrm{D}$, white arrowheads). Also note the development of a large, well-defined subchondral cystic lesion underneath the full-thickness defect $(\mathrm{C}+\mathrm{D}$, white arrow). 

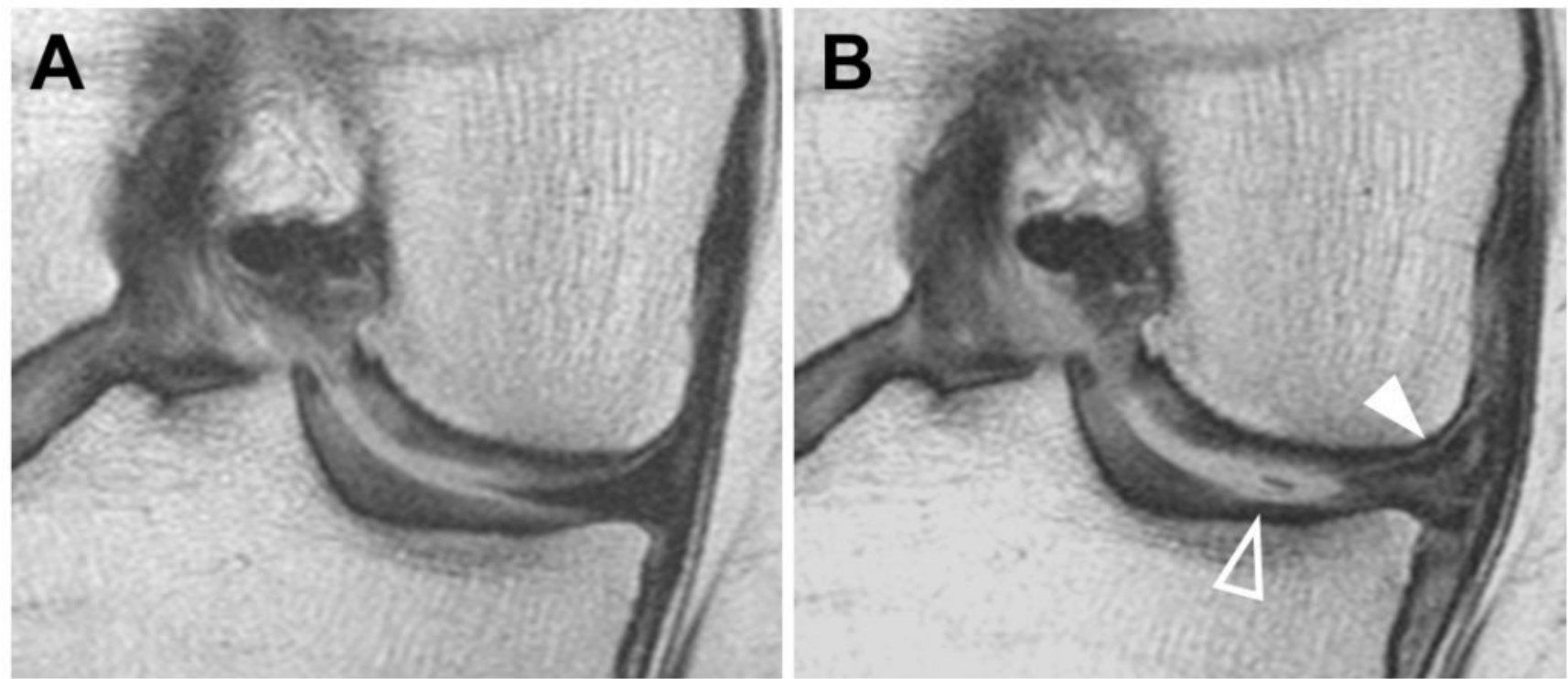

Fig 4.

MRI of the right knee of a 62-year old man with diabetes with coronal PD-weighted sequences without fat suppression at baseline (A) and 48-month follow-up (B). The baseline study (A) shows a normal medial meniscus without any intrasubstance abnormalities. In the 48-month follow-up study (B) meniscal extrusion with a newly developed multidirectional medial meniscus tear are shown (B, white arrowhead). Furthermore, cartilage thinning in the center of the medial tibia is demonstrated in the 48-month follow-up scan (hollow white arrowhead). 
Table 1.

Participant demographics

\begin{tabular}{|c|c|c|c|c|}
\hline & All $(n=488)$ & Diabetics (n=244) & $\begin{array}{l}\text { Diabetes-free controls } \\
\quad(n=244)\end{array}$ & p-values \\
\hline \multicolumn{5}{|l|}{ Demographics at baseline } \\
\hline Age (years) & $63.14 \pm 9.09$ & $63.00 \pm 8.97$ & $63.28 \pm 9.22$ & 0.73 \\
\hline Body mass index $\left(\mathrm{kg} / \mathrm{m}^{2}\right)$ & $31.28 \pm 4.54$ & $31.54 \pm 4.61$ & $31.02 \pm 4.47$ & 0.20 \\
\hline Females $[\mathrm{n}(\%)] /$ Males $[\mathrm{n}(\%)]$ & $256(52.5 \%) / 232(47.5 \%)$ & $127(52.0 \%) / 117(48.0 \%)$ & $129(52.9 \%) / 115(47.1 \%)$ & 0.85 \\
\hline Right knee Kellgren-Lawrence & & & & 0.99 \\
\hline Grade $0[\mathrm{n}(\%)]$ & $198(40.6 \%)$ & $99(40.6 \%)$ & $99(40.6 \%)$ & \\
\hline Grade 1 [n (\%)] & $117(24.0 \%)$ & $59(24.2 \%)$ & $58(23.8 \%)$ & \\
\hline Grade $2[\mathrm{n}(\%)]$ & $173(35.4 \%)$ & $86(35.2 \%)$ & $87(35.7 \%)$ & \\
\hline PASE & $148.77 \pm 82.11$ & $144.59 \pm 80.41$ & $152.95 \pm 83.73$ & 0.26 \\
\hline History of knee Injury [n (\%)] & $179(33.7 \%)$ & $91(37.3 \%)$ & $88(36.1 \%)$ & 0.75 \\
\hline History of knee surgery [n (\%)] & $64(13.1 \%)$ & $35(14.3 \%)$ & $29(11.9 \%)$ & 0.46 \\
\hline Racial composition & & & & $<0.001$ \\
\hline Caucasian $[\mathrm{n}(\%)]$ & $350(71.7 \%)$ & $146(59.8 \%)$ & $204(83.6 \%)$ & \\
\hline African American [n (\%)] & $122(25.0 \%)$ & $87(35.7 \%)$ & $35(14.3 \%)$ & \\
\hline Asian $[\mathrm{n}(\%)]$ & $6(1.2 \%)$ & $5(2.0 \%)$ & $1(0.4 \%)$ & \\
\hline Other Non-white [n (\%)] & $10(2.0 \%)$ & $6(2.5 \%)$ & $4(1.6 \%)$ & \\
\hline
\end{tabular}

Table shows the participant demographics at baseline. Continues data are expressed as mean $\pm \mathrm{SD}$. Categorical data are presented in numbers of participants with percentage in parentheses. P-values listed in the right column refer to the differences between diabetics and diabetes-free controls and were calculated using either Pearson's $\chi 2$-test or independent samples t-test as appropriate. 
Table 2.

WORMS - Cross sectional differences of WORMS findings in diabetics and diabetes-free controls at baseline

\begin{tabular}{|c|c|c|c|c|c|}
\hline \multirow[b]{2}{*}{ Baseline WORMS parameters } & \multicolumn{2}{|c|}{ Diabetics } & \multicolumn{2}{|c|}{ Diabetes-free controls } & \multirow{2}{*}{ p-values } \\
\hline & Adjusted means & {$[95 \% \mathrm{CI}]$} & Adjusted means & {$[95 \% \mathrm{CI}]$} & \\
\hline \multicolumn{6}{|l|}{ Overall } \\
\hline WORMS overall sum score & 11.60 & {$[10.54,12.66]$} & 11.35 & {$[10.29,12.40]$} & 0.73 \\
\hline \multicolumn{6}{|l|}{ Cartilage } \\
\hline Global sum score & 5.53 & {$[4.96,6.11]$} & 5.43 & {$[4.88,5.98]$} & 0.79 \\
\hline Patella & 2.20 & {$[1.95,2.45]$} & 2.13 & {$[1.90,2.36]$} & 0.68 \\
\hline Trochlea & 1.62 & {$[1.41,1.83]$} & 1.57 & {$[1.35,1.80]$} & 0.77 \\
\hline Medial tibia & 0.16 & {$[0.08,0.23]$} & 0.15 & {$[0.07,0.24]$} & 0.97 \\
\hline Lateral tibia & 0.52 & {$[0.39,0.65]$} & 0.52 & {$[0.38,0.66]$} & 0.98 \\
\hline Medial femur & 0.63 & {$[0.49,0.76]$} & 0.70 & {$[0.55,0.86]$} & 0.45 \\
\hline Lateral femur & 0.41 & {$[0.29,0.53]$} & 0.36 & {$[0.24,0.48]$} & 0.59 \\
\hline \multicolumn{6}{|l|}{ Meniscus } \\
\hline Meniscus sum score & 1.95 & {$[1.66,2.23]$} & 1.66 & {$[1.38,1.94]$} & 0.17 \\
\hline Medial meniscus & 1.08 & {$[0.90,1.26]$} & 0.92 & {$[0.77,1.08]$} & 0.19 \\
\hline Lateral meniscus & 0.87 & {$[0.66,1.07]$} & 0.74 & {$[0.53,0.95]$} & 0.41 \\
\hline \multicolumn{6}{|l|}{ Other parameters } \\
\hline Global BME score & 1.73 & {$[1.49,1.96]$} & 1.93 & {$[1.68,2.19]$} & 0.24 \\
\hline Global subarticular cyst score & 1.32 & {$[1.13,1.52]$} & 1.22 & {$[0.99,1.45]$} & 0.53 \\
\hline Global ligament score & 0.36 & {$[0.23,0.50]$} & 0.43 & {$[0.28,0.59]$} & 0.53 \\
\hline Effusion & 0.20 & {$[0.14,0.26]$} & 0.15 & {$[0.10,0.20]$} & 0.22 \\
\hline Popliteal cyst & 0.50 & {$[0.41,0.59]$} & 0.45 & {$[0.35,0.55]$} & 0.42 \\
\hline
\end{tabular}

Data are given as adjusted means, corrected for race, age, sex, baseline BMI, baseline K/L score, with [95\% confidence intervals] and computed as the numerical means for both groups at baseline. 
Table 3.

WORMS - Longitudinal changes of WORMS findings in diabetics and diabetes-free controls over 48 months

\begin{tabular}{|c|c|c|c|c|c|}
\hline \multirow[b]{2}{*}{ Changes in WORMS parameters } & \multicolumn{2}{|c|}{ Diabetics } & \multicolumn{2}{|c|}{ Diabetes-free controls } & \multirow{2}{*}{ p-values } \\
\hline & Adjusted means & {$[95 \% \mathrm{CI}]$} & Adjusted means & {$[95 \% \mathrm{CI}]$} & \\
\hline \multicolumn{6}{|l|}{ Overall } \\
\hline WORMS overall sum score & 4.87 & {$[4.17,5.57]$} & 3.23 & {$[2.60,3.85]$} & 0.001 \\
\hline \multicolumn{6}{|l|}{ Cartilage } \\
\hline Global sum score & 2.57 & {$[2.22,2.93]$} & 1.52 & {$[1.23,1.82]$} & $<0.001$ \\
\hline Patella & 0.60 & {$[0.48,0.73]$} & 0.35 & {$[0.24,0.45]$} & 0.005 \\
\hline Trochlea & 0.53 & {$[0.38,0.68]$} & 0.38 & {$[0.27,0.50]$} & 0.14 \\
\hline Medial tibia & 0.24 & {$[0.14,0.34]$} & 0.19 & {$[0.09,0.29]$} & 0.42 \\
\hline Lateral tibia & 0.32 & {$[0.22,0.43]$} & 0.18 & {$[0.11,0.26]$} & 0.03 \\
\hline Medial femur & 0.45 & {$[0.33,0.57]$} & 0.24 & {$[0.13,0.34]$} & 0.01 \\
\hline Lateral femur & 0.43 & {$[0.30,0.55]$} & 0.18 & {$[0.11,0.25]$} & 0.001 \\
\hline \multicolumn{6}{|l|}{ Meniscus } \\
\hline Meniscus sum score & 1.26 & {$[0.97,1.54]$} & 0.62 & {$[0.37,0.87]$} & 0.001 \\
\hline Medial meniscus & 0.71 & {$[0.52,0.91]$} & 0.37 & {$[0.20,0.55]$} & 0.01 \\
\hline Lateral meniscus & 0.54 & {$[0.36,0.72]$} & 0.25 & {$[0.10,0.40]$} & 0.01 \\
\hline \multicolumn{6}{|l|}{ Other parameters } \\
\hline Global BME score & 0.49 & {$[0.28,0.69]$} & 0.53 & {$[0.35,0.71]$} & 0.75 \\
\hline Global subarticular cyst score & 0.27 & {$[0.11,0.43]$} & 0.36 & {$[0.20,0.52]$} & 0.45 \\
\hline Global ligament score & 0.11 & {$[0.00,0.21]$} & 0.02 & {$[-0.06,0.11]$} & 0.28 \\
\hline Effusion & 0.13 & {$[0.05,0.21]$} & 0.08 & {$[0.01,0.15]$} & 0.40 \\
\hline Popliteal cyst & 0.02 & {$[0.04,0.09]$} & 0.03 & {$[-0.06,0.12]$} & 0.88 \\
\hline
\end{tabular}

Data are given as adjusted means, corrected for race, age, sex, baseline BMI, baseline K/L score, with [95\% confidence intervals] and computed as the absolute change between baseline and 48-months. P-values showing statistical significance $(<0.05)$ are in bold. 
Table 4.

WORMS - Longitudinal changes of WORMS findings in severe-diabetics, non-severe-diabetics, and diabetes-free controls over 48 months

\begin{tabular}{|c|c|c|c|c|c|c|c|}
\hline \multirow{2}{*}{$\begin{array}{l}\text { Changes in } \\
\text { WORMS } \\
\text { parameters }\end{array}$} & \multicolumn{2}{|c|}{ Severe-diabetics } & \multicolumn{2}{|c|}{ Diabetes-free controls } & \multirow[b]{2}{*}{ p-values* } & \multicolumn{2}{|c|}{ Non-severe-diabetics } \\
\hline & $\begin{array}{c}\text { Adjusted } \\
\text { means }\end{array}$ & {$[95 \% \mathrm{CI}]$} & $\begin{array}{c}\text { Adjusted } \\
\text { means }\end{array}$ & {$[95 \% \mathrm{CI}]$} & & $\begin{array}{l}\text { Adjusted means [95\% } \\
\text { CI] }\end{array}$ & p-values *** \\
\hline \multicolumn{8}{|l|}{ Overall } \\
\hline WORMS overall sum score & 5.83 & {$[4.52,7.14]$} & 3.21 & {$[2.60,3.82]$} & $<0.001$ & $4.21[3.33,5.09]$ & 0.06 \\
\hline \multicolumn{8}{|l|}{ Cartilage } \\
\hline Global sum score & 3.01 & {$[2.34,3.69]$} & 1.50 & {$[1.21,1.78]$} & $<0.001$ & $2.34[1.93,2.75]$ & 0.12 \\
\hline Patella & 0.70 & {$[0.46,0.94]$} & 0.34 & {$[0.24,0.45]$} & 0.011 & $0.57[0.41,0.72]$ & 0.55 \\
\hline Trochlea & 0.49 & {$[0.27,0.70]$} & 0.40 & {$[0.28,0.51]$} & 0.450 & $0.54[0.35,0.73]$ & 0.81 \\
\hline Medial tibia & 0.32 & {$[0.14,0.50]$} & 0.18 & {$[0.08,0.28]$} & 0.180 & $0.20[0.08,0.32]$ & 0.26 \\
\hline Lateral tibia & 0.35 & {$[0.17,0.52]$} & 0.18 & {$[0.11,0.25]$} & 0.077 & $0.33[0.17,0.50]$ & 0.94 \\
\hline Medial femur & 0.55 & {$[0.35,0.75]$} & 0.22 & {$[0.12,0.33]$} & 0.004 & $0.38[0.24,0.52]$ & 0.13 \\
\hline Lateral femur & 0.61 & {$[0.35,0.87]$} & 0.18 & {$[0.10,0.25]$} & 0.002 & $0.31[0.17,0.45]$ & 0.09 \\
\hline \multicolumn{8}{|l|}{ Meniscus } \\
\hline Meniscus sum score & 1.50 & {$[0.99,2.01]$} & 0.63 & {$[0.39,0.87]$} & 0.004 & $1.02[0.70,1.34]$ & 0.11 \\
\hline Medial meniscus & 0.75 & {$[0.43,1.08]$} & 0.39 & {$[0.22,0.56]$} & 0.062 & $0.61[0.39,0.83]$ & 0.36 \\
\hline Lateral meniscus & 0.74 & {$[0.41,1.08]$} & 0.24 & {$[0.10,0.39]$} & 0.011 & $0.41[0.17,0.64]$ & 0.15 \\
\hline \multicolumn{8}{|l|}{ Other parameters } \\
\hline Global BME score & 0.67 & {$[0.34,1.00]$} & 0.53 & {$[0.36,0.71]$} & 0.443 & $0.37[0.11,0.63]$ & 0.21 \\
\hline Global subarticular cyst score & 0.29 & {$[0.07,0.51]$} & 0.36 & {$[0.20,0.52]$} & 0.624 & $0.26[0.04,0.48]$ & 0.79 \\
\hline Global ligament score & 0.11 & {$[-0.01,0.23]$} & 0.03 & {$[-0.05,0.11]$} & 0.314 & $0.10[-0.02,0.22]$ & 0.99 \\
\hline Effusion & 0.20 & {$[0.09,0.31]$} & 0.08 & {$[0.01,0.15]$} & 0.106 & $0.08[-0.03,0.19]$ & 0.12 \\
\hline Popliteal cyst & 0.02 & {$[-0.06,0.09]$} & 0.03 & {$[-0.06,0.11]$} & 0.874 & $0.03[-0.07,0.13]$ & 0.45 \\
\hline
\end{tabular}

Data are given as adjusted means, corrected for race, age, sex, baseline BMI, baseline K/L score, with [95\% confidence intervals] and computed as the absolute change between the 48 -months and the baseline. P-values showing statistical significance $(<0.05)$ are shown in bold characters.

= p-values indicate differences between severe-diabetics and diabetes-free controls; The column on the far right shows the adjusted means for diabetics without severe disease and

**

p-values indicate the differences when compared to diabetics with severe disease. 
Table 5.

WORMS - Longitudinal changes of WORMS findings in non-severe-diabetics and diabetes-free controls over 48 months

\begin{tabular}{lccccc}
\hline \multicolumn{1}{c}{$\begin{array}{c}\text { Nhanges } \text { in WORMS } \\
\text { parameters }\end{array}$} & Adjusted means & {$[\mathbf{9 5 \%}$ CI] } & Adjusted means & {$[\mathbf{9 5 \%}$ CI] } & \\
\hline Overall & & & & & p-values* \\
\hline WORMS overall sum score & 4.39 & {$[3.53,5.25]$} & 3.24 & {$[2.63,3.86]$} & $\mathbf{0 . 0 3 6}$ \\
\hline Cartilage & & & & & \\
\hline Global sum score & 2.33 & {$[1.94,2.73]$} & 1.52 & {$[1.23,1.81]$} & $\mathbf{0 . 0 0 2}$ \\
Patella & 0.56 & {$[0.40,0.71]$} & 0.35 & {$[0.24,0.45]$} & $\mathbf{0 . 0 3 5}$ \\
Trochlea & 0.54 & {$[0.34,0.74]$} & 0.38 & {$[0.27,0.50]$} & 0.208 \\
Medial tibia & 0.20 & {$[0.08,0.33]$} & 0.18 & {$[0.08,0.28]$} & 0.795 \\
Lateral tibia & 0.30 & {$[0.16,0.45]$} & 0.18 & {$[0.11,0.25]$} & 0.145 \\
Medial femur & 0.39 & {$[0.25,0.53]$} & 0.24 & {$[0.14,0.34]$} & 0.107 \\
Lateral femur & 0.61 & {$[0.35,0.87]$} & 0.18 & {$[0.10,0.25]$} & $\mathbf{0 . 0 0 2}$ \\
\hline Meniscus & & & & & \\
\hline Meniscus sum score & 1.13 & {$[0.79,1.47]$} & 0.64 & {$[0.39,0.88]$} & $\mathbf{0 . 0 2 2}$ \\
Medial meniscus & 0.69 & {$[0.45,0.93]$} & 0.39 & {$[0.22,0.56]$} & 0.053 \\
Lateral meniscus & 0.44 & {$[0.22,0.66]$} & 0.25 & {$[0.10,0.40]$} & 0.134 \\
\hline Other parameters & & & & & \\
\hline Global BME score & 0.39 & {$[0.13,0.66]$} & 0.52 & {$[0.34,0.70]$} & 0.463 \\
Global subarticular cyst score & 0.26 & {$[0.04,0.49]$} & 0.36 & {$[0.21,0.52]$} & 0.496 \\
Global ligament score & 0.11 & {$[-0.02,0.24]$} & 0.03 & {$[-.005,0.11]$} & 0.345 \\
Effusion & 0.10 & {$[0.01,0.21]$} & 0.09 & {$[0.016,0.16]$} & 0.901 \\
Popliteal cyst & 0.04 & {$[-0.06,0.14]$} & 0.03 & {$[-0.05,0.12]$} & 0.891 \\
\hline & & & & & \\
\hline
\end{tabular}

Data are given as adjusted means, corrected for race, age, sex, baseline BMI, baseline K/L score, with [95\% confidence intervals] and computed as the absolute change between the 48 -months and the baseline. P-values showing statistical significance $(<0.05)$ are shown in bold characters. 